$\mathrm{B}_{\mathrm{P}}$ рожАенный булАезный эпидермолиз: современные методы диагностики и терапии. Перспективы регенеративной медицины

Кубанов А. А., Карамова А. Э. ${ }^{*}$, Мончаковская Е. С.

ФГБУ «Государственный научный центр дерматовенерологии и косметологии» Минздрава России 107076, Россия, г. Москва, ул. Короленко, д. 3, стр. 6

Врожденный буллезный эпидермолиз - клинически и генетически гетерогенная группа наследственных заболеваний кожи, характеризующаяся образованием пузырей и/или эрозий в ответ на незначительное механическое воздействие. Многообразие и тяжесть клинических проявлений заболевания определяют раннюю инвалидизацию больных и снижение качества жизни, что требует разработки патогенетических и этиологических методов лечения. Методы генной терапии представляют собой наиболее перспективное направление для изучения, поскольку они способны воздействовать на причину развития врожденного буллезного эпидермолиза.

Ключевые слова: врожденный буллезный эпидермолиз, клеточная терапия, генная терапия, редактирование генома, вирусные векторы.

Конфрликт интересов: авторы заявляют об отсутствии потенциального конфрликта интересов, требующего раскрытия в данной статье.

Для цитирования: Кубанов А. А., Карамова А. Э., Мончаковская Е. С. Врожденный буллезный эпидермолиз: современные методы диагностики и терапии. Перспективы регенеративной медицины. Вестник дерматологии и венерологии. 2020; 96 (1): 10-17. https://doi.org/10.25208/vdv551-2020-96-1-10-17 


\title{
Congenital epidermolysis bullosa: modern methods of diagnosis and therapy. Prospects for regenerative medicine
}

\author{
Alexey A. Kubanov, Arfenya E. Karamova*, Ekaterina S. Monchakovskaya
}

State Research Center of Dermatovenereology and Cosmetology, Ministry of Health of the Russian Federation Korolenko str., 3, bldg 6, Moscow, 107076, Russia

Congenital epidermolysis bullosa is a clinically and genetically heterogenous group of hereditary skin diseases characterized by the formation of bullae and/or erosions in response to insignificant mechanical effect. The variety and severity of clinical manifestations of the disease determine the early disablement of patients and the decrease in the quality of life, which requires the development of pathogenetic and etiological methods of treatment. Methods of gene therapy are the most promising direction to study, since they can affect the cause of congenital epidermolysis bullosa.

Keywords: congenital epidermolysis bullosa, cell therapy, gene therapy, genome editing, viral vectors.

Conflict of interest: the authors state that there is no potential conflict of interest requiring disclosure in this article.

For citation: Alexey A. Kubanov, Arfenya E. Karamova, Ekaterina S. Monchakovskaya. Congenital epidermolysis bullosa: modern methods of diagnosis and therapy. Prospects for regenerative medicine. Vestnik Dermatologii i Venerologii. 2020; 96 (1): 10-17. https://doi.org/10.25208/vdv551-2020-96-1-10-17 


\section{Определение и эпидемиология}

Врожденный буллезный эпидермолиз (ВБЭ) группа наследственных заболеваний кожи, обусловленных мутациями в генах структурных белков эпидермиса и дермо-эпидермального соединения. Основной клинический признак ВБЭ - образование пузырей и/или эрозий на коже и слизистых оболочках в ответ на незначительную механическую травму [1].

ВБЭ относится к орфанным заболеваниям. По данным Национальных институтов здоровья (NIH, США), орфанным считается заболевание, число случаев которого составляет менее 200000 на территории США [2]. В Российской Федерации, согласно Федеральному закону №323-Ф3 (ред. от 25.06.2012) «Об основах охраны здоровья граждан в Российской Федерации», в котором законодательно введено понятие редких (орфанных) болезней и согласно которому орфанными считаются заболевания с распространенностью не более 10 случаев заболевания на 100000 населения [3].

Для изучения заболеваемости и распространенности ВБЭ и упрощения сбора информации во многих странах были созданы регистры больных, страдающих ВБЭ [4-9].

В Российской Федерации изучение распространенности и заболеваемости ВБЭ ведется с 2014 г. [10]. По состоянию на конец 2016 г. в 60 субъектах Российской Федерации число зарегистрированных заболеваний ВБЭ составило 417. Соответствующий показатель распространенности ВБЭ равен 3,9 на 1 млн населения. Заболеваемость ВБЭ всего населения Российской Федерации в 2016 г. составила 13,90 на 1 млн населения [11].

\section{Классификация и клиническая картина}

Согласно классификации ВБЭ от 2014 г., в зависимости от уровня образования пузыря выделяют 4 основных типа заболевания: простой буллезный эпидермолиз (ПБЭ), пограничный буллезный эпидермолиз (ПгрБЭ), дистрофический буллезный эпидермолиз (ДБЭ) и синдром Киндлера [1].

ВБЭ - клинически и генетически гетерогенная группа заболеваний, которые возникают вследствие мутаций в генах структурных белков эпидермиса и дермо-эпидермального соединения [12].

В настоящее время выявлены 20 генов, приводящих к развитию ВБЭ [13]; в 2016 г. был выявлен новый структурный белок, участвующий в патогенезе ВБЭ. Установлено, что мутация в гене белка KLHL24 (Kelch-подобный белок 24) отвечает за развитие нового клинического подтипа ПБЭ [14-16]. Вследствие мутаций в данном белке возникает излишнее убиквитинирование и деградация кератина 14, который является компонентом промежуточных фриламентов цитоскелета клетки [17].

Кроме того, новые клинические френотипы были выявлены благодаря обнаружению мутаций в генах CD151, PLEC1a, DST, EXPH5 и ITGA3 [18-33].

Вовлеченность большого числа генов в патогенез заболевания определяет многообразие клинических проявлений. Распространенность поражения варьирует от локализованных высыпаний, ограниченных кожей ладоней и подошв, до генерализованных высыпаний С вовлечением слизистых оболочек внутренних органов. К одному из самых прогностически неблагопри- ятных клинических подтипов ВБЭ относится тяжелый генерализованный рецессивный дистрофический буллезный эпидермолиз (РДБЭ) [34]. Он характеризуется множественными пузырями и эрозивно-язвенными дефектами, которые постоянно возникают спонтанно и/ или вследствие травмы и приводят к прогрессирующему рубцеванию и развитию распространенных очагов рубцовой атрофии, псевдосиндактилий, деформации кистей по типу «варежки», контрактур суставов [35]. Тяжелый генерализованный РДБЭ сопровождается отставанием в фризическом развитии, анемией, нутритивной недостаточностью, ранним развитием осложнений и ранней смертностью [36-39].

Избыточное образование рубцовой ткани и наличие длительно незаживающих дефектов кожи приводят к развитию плоскоклеточного рака кожи (ПРК) [40, 41]. Для ПРК при ВБЭ характерны быстрый рост опухоли, быстрое метастазирование и возникновение множественных очагов первичной опухоли [41]. ПРК является ведущей причиной смертности среди больных рецессивным дистрофическим буллезным эпидермолизом. Кумулятивный риск развития ПРК у больных РДБЭ с тяжелым генерализованным подтипом составляет:

- 7,5\% в возрасте 20 лет;

- 26,7\% в возрасте 25 лет;

- 51,7\% в возрасте 30 лет;

- 90,1\% в возрасте 55 лет [40].

5-летняя выживаемость среди больных с РДБЭассоциированным ПРК практически равна 0\% [40, 41].

\section{Методы диагностики}

Учитывая многообразие клинических проявлений ВБЭ и сложность клинической диагностики, для подтверждения диагноза необходимы лабораторноинструментальные методы исследования. С целью подтверждения диагноза ВБЭ применяют иммунофрлюоресцентное антигенное картирование (ИАК), трансмиссионную электронную микроскопию (ТЭМ) и молекулярно-генетическую диагностику.

ТЭМ и ИАК позволяют выявить уровень расщепления в биоптатах кожи больных для дифференциальной диагностики между основными типами ВБЭ [1]. Методы генетической диагностики позволяют определить мутации в генах структурных белков дермо-эпидермального соединения, соответствующие определенным клиническим подтипам ВБЭ [42].

Трансмиссионная электронная микроскопия

ТЭМ позволяет визуализировать специфические ультраструктуры (кератиновые фриламенты, десмосомы, полудесмосомы, супрабазальные плотные пластинки, якорные фрибриллы и фриламенты) и дать их количественную характеристику, характерную для определенных клинических подтипов ВБЭ [43]. Однако в настоящее время ТЭМ применяется редко, так как представляет собой трудоемкий метод, требующий наличия дорогостоящего оборудования и обученного персонала с большим опытом проведения исследования для диагностики ВБЭ $[42,43]$.

Иммуносрлюоресцентное антигенное картирование

Иммунофлюоресцентное антигенное картирование (ИАК) позволяет определить не только уровень формирования пузыря, но и дефицит структурных белков эпидермиса и дермо-эпидермального соединения [44]. Установление клинического подтипа ВБЭ становится возможным при выявлении отсутствия или де- 
фрицита определенного белка. Полученная информация о нарушениях экспрессии белков кожи позволяет ограничить количество генов, которые необходимо изучить в ходе последующего молекулярно-генетического исследования [1].

\section{Молекулярно-генетическое исследование}

Молекулярно-генетическое исследование рекомендуется проводить всем пациентам с ВБЭ, поскольку определение мутации и типа наследования необходимо для прогнозирования течения заболевания, планирования семьи, пренатальной диагностики и для назначения персонализированных методов терапии [45].

Выявление мутаций имеет ценное значение для накопления научных данных о заболевании, поскольку в последнее время возросло число новых ранее не описанных клинических френотипов [46]. Точные знания о характере и локализации мутации также необходимы для создания клеточных культур, используемых в исследованиях, и для разработки методов генной терапии.

\section{Методы терапии врожденного буллезного эпидермолиза}

В настоящее время патогенетические методы лечения ВБЭ находятся на разных этапах клинических исследований. Применяемое в настоящее время лечение является симптоматическим и направлено на ускорение заживления эрозивно-язвенных дефектов кожи и слизистых оболочек внутренних органов, предотвращение развития вторичной инфекции и коррекцию осложнений и сопутствующей патологии [47].

Наружная терапия - основной метод лечения. Она заключается в обработке пузырей и эрозий/язв растворами антисептиков, регулярных перевязках с применением атравматичных неадгезивных перевязочных средств [48]. Больным также назначается лечение для купирования боли и зуда, которые препятствуют нормальному заживлению и значительно снижают качество жизни пациентов $[49,50]$. При наличии железодефицитной анемии, которая часто встречается при тяжелых протекающих подтипах ПгрБЭ и ДБЭ, показано назначение препаратов железа, желательно в парентеральной форме [51].

У больных ВБЭ с поражением слизистой оболочки полости рта/глотки/пищевода наблюдается недостаточность питания [52], поэтому данной категории больных назначается нутритивная поддержка высокобелковыми и высокоэнергетическими смесями [53].

При развитии у больных ВБЭ вторичного инфиццирования применяются повязки с содержанием противомикробных и антисептических средств, системные и топические антибактериальные препараты [47, 48, 54].

\section{Патогенетические методы терапии ВБЭ}

Среди современных и перспективных методов лечения ВБЭ выделяют белковую, клеточную и генную терапию [55]. Белковая терапия заключается во внутривенном или внутриочаговом введении в отдельные участки кожи белка, который у пациента либо отсутствует, либо является дефектным [56]. Рекомбинантный белок вводится внутривенно или внутрикожно. Проведенные доклинические исследования белковой терапии продемонстрировали перспективные результаты, однако для лечения пациентов требуется дальнейшее изучение и совершенствование этого метода [57].

\section{Клеточная терапия}

Клеточная терапия заключается во введении в организм человека аутологичных или аллогенных клеточных культур, способных заменить фрункцию генетически поврежденных клеток [58]. Цель клеточной терапии при дистрофическом буллезном эпидермолизе - заживление дефектов кожи и предупреждение их образования в последующем за счет повышения количества коллагена VII типа в зоне базальной мембраны [59]. Основным источником коллагена VII типа в коже являются фрибробласты и кератиноциты [60]. Фибробласты являются простыми для культивирования, сохраняют в культуре диплоидный кариотип, имеют низкую экспрессию антигенов гистосовместимости после пассирования и характеризуются отсутствием онкогенного потенциала, что позволяет использовать культивируемые in vitro фрибробласты человека в терапевтических целях [61, 62]. Низкая иммуногенность позволяет использовать для пересадки аллогенные фрибробласты, полученные от неродственного пациенту донора. В отношении аллогенных фрибробластов существует возможность быстрого накопления большого количества относительно недорогого клеточного материала, его криоконсервации и хранения больших запасов клеток для их срочного применения [62].

В нескольких клинических исследованиях I и II фразы с небольшим числом участников оценена эффективность и безопасность терапии больных РДБЭ методом внутрикожного введения аллогенных фибробластов [59, 63-65]. Использовали препарат аллогенных фиибробластов, содержащий $20 \times 10^{6}$ клеток в 1 мл $[64,65]$. Введение фрибробластов осуществляли однократно в различные участки: в интактный участок кожи без пузыря, в дно эрозий или по краям эрозий. Было обнаружено, что внутрикожные инъекции фрибробластов приводят к повышению экспрессии коллагена VII типа, увеличению количества якорных фибрилл в зоне дермоэпидермального соединения и ускорению заживления эрозий и язв. Серьезных нежелательных явлений зарегистрировано не было [59, 63-65].

\section{Введение гемопоэтических стволовых клеток}

Впервые трансплантация костного мозга на модели лабораторных мышей с РДБЭ была проведена в работе Tolar J. и соавт. в 2009 г. [66]. В результате проведенного исследования было отмечено увеличение экспрессии коллагена VII типа и возникновение рудиментарных якорных фрибрилл в зоне базальной мембраны в области имеющихся дефектов кожи, что указывает на то, что часть гемопоэтических стволовых клеток (ГСК) способна участвовать в процессе заживления [66]. Данная работа стала основой для клинических исследований с применением ГСК у больных ВБЭ [67].

В 2010 г. в публикации Wagner J. и соавт. впервые описана процедура трансплантации аллогенного костного мозга 6 больным РДБЭ. Это исследование с применением системной клеточной терапии у больных РДБЭ показало, что донорские клетки мигрируют к местам повреждения и эффект поддерживается на протяжении длительного времени. В результате проведенного лечения у 5 больных наблюдалось значительное уменьшение образования пузырей, улучшение заживления, повышение экспрессии коллагена VII типа и образование якорных фибрилл. Существенным недостатком процедуры является тяжелая переносимость в связи с проведением полной миелоабляции. 
Результаты данной работы также предполагают использование генетически скорректированных аутологичных ГСК [68].

Введение мезенхимальных стволовых клеток

По данным Sasaki M. Et al. (2008), мезенхимальные стволовые клетки (МСК) играют роль в заживлении ран, которая продемонстрирована на лабораторных животных. Это обусловлено мультипотентностью МСК, которая позволяет им дифференцироваться в различные виды клеток, в том числе и в фибробласты [69]. Petrof и соавт. (2015) провели исследование с внутривенным введением аллогенных МСК 10 детям с РДБЭ. Клинически отмечалось уменьшение тяжести проявлений заболеваний и улучшение качества жизни пациентов [70]. Недостатком аллогенной трансплантации клеточных культур является поиск доноров. Однако благодаря получению индуцированных плюрипотентных стволовых клеток (иПСК) [71, 72] появилась возможность применения аутологичной клеточной трансплантации при ВБЭ. Эфрфективность данного метода была продемонстрирована в исследованиях с моделями серповидноклеточной анемии, дистрофии Лейдена и дегенерации макулы [73-75]. В исследовании с лабораторными мышами после трансплантации иПСК терминально дифференцированные клетки кожи и костного мозга практически не вызвали иммунного ответа [76]. У больных РДБЭ и ПгрБЭ также были выделены иПСК, что может стать основой для создания персонализированной клеточной терапии $[77,78]$.

Все проведенные клинические исследования с применением различных клеточных культур продемонстрировали хорошие результаты в виде улучшения эпителизации эрозивно-язвенных дефектов кожи и повышения экспрессии коллагена VII типа в зоне базальной мембраны. Однако к существенному недостатку процедур относится кратковременность эффректа.

Генная терапия

Наиболее распространенный метод генной терапии - применение вирусных векторов. Для лечения РДБЭ были разработаны аденовирусные, ретровирусные и лентивирусные векторы [79]. Ретровирусные векторы использовали для трансдукции фрибробластов, которые затем вводили в мышиную модель РДБЭ. В результате исследования продемонстрировано, что модифицированные фрибробласты способны увеличивать экспрессию коллагена VII типа и образовывать функционально активные якорные фибриллы, и как следствие, приводить к клиническому улучшению $[79,80]$. B работах Gache Y., Baldeschi C., Del Rio M. et al. (2001) и Titeux M., Pendaries V., Zanta-Boussif M. A. et al. (2010) из модифицированных клеток от больного РДБЭ были выращены кожные лоскуты [80, 81]. Впервые ретровирусные векторы в лечении РДБЭ были использованы для трансдукции кератиноцитов, содержащих человеческий ген COL7A1 [81]. Из модифицированных кератиноцитов затем были выращены эпидермальные лоскуты для аутологичной терапии. После проведенного лечения клинический эффект наблюдался в течение 12 месяцев.

Аденовирусные векторы также использовались для коррекции мутаций в кератиноцитах и фрибробластах больных РДБЭ. Кроме того, эти клетки затем были преобразованы в иПСК [82]. Скорректированные иПСК дифреренцировались в кератиноциты, способные продуцировать нормальный коллаген VII типа и образовывать все слои эпидермиса. Модифицированные фибробласты, полученные с помощью лентивирусных векторов, в эксперименте показали свою способность экспрессировать коллаген VII типа в кожных лоскутах иммунодефицитных мышей [83].

В 2017 г. в работе Hirsch et. al впервые была проведена трансплантация эпидермальных лоскутов на 80\% поверхности туловища пациенту 7 лет, с тяжелым генерализованным ПгрБЭ. Эпидермальные лоскуты содержали генетически модицифированные кератиноциты, полученные из биоптатов кожи больного. Культура кератиноцитов с мутацией в гене LAMB3 была скорректирована с применением ретровирусных векторов. В течение 3-летнего периода наблюдения после терапии у пациента не наблюдалось появления пузырей или эрозий даже после механического воздействия, также был восстановлен тургор кожи. В биоптатах кожи, полученных после трансплантации, экспрессия ламинина-332 соответствовала уровню экспрессии в биоптатах кожи здоровых добровольцев [84].

\section{Заключение}

В настоящее время результаты экспериментальных и клинических исследований с применением различных методов белковой, клеточной и генной терапии свидетельствуют о возможности увеличения содержания структурных белков в зоне дермо-эпидермального соединения и клинической эффективности в виде сокращения сроков заживления длительно существующих дефектов кожи у больных буллезным эпидермолизом. Методы генной терапии вызывают наибольший интерес исследователей, поскольку они воздействуют на причину развития ВБЭ.

Дальнейшие разработка и совершенствование данных методов позволят улучшить качество жизни таких пациентов, отсрочить развитие плоскоклеточного рака кожи и увеличить продолжительность жизни больных ВБЭ.

\section{Литература/References}

1. Fine J.D., Bruckner-Tuderman L., Eady R.A. et al. Inherited epidermolysis bullosa: Updated recommendations on diagnosis and classification. J Am Acad Dermatol 2014; 70: 1103-1126.

2. https://report.nih.gov/nihfactsheets/ViewFactSheet.aspx?csid=126

3. Федеральный закон от 21.11.2011 №323-Ф3 (ред. от 25.06.2012) «0б основах охраны здоровья граждан в Российской Федерации». [Fed- eral Law of November 21, 2011 No. 323-FZ (as amended on June 25, 2012) "On the Basics of Protecting the Health of Citizens in the Russian Federation".]

4. Fine J.D. Epidemiology of inherited epidermolysis bullosa based on incidence and prevalence estimates from the National Epidermolysis Bullosa Registry. JAMA Dermatol 2016; 152 (11): 1231. 
5. Danescu S., Has C., Senila S. et al. Epidemiology of inherited epidermolysis bullosa in Romania and genotype-phenotype correlations in patients with dystrophic epidermolysis bullosa. J Eur Acad Dermatol Venereol 2015; 29 (5): 899—903.

6. Pavicić Z., Kmet-Vizintin P., Kansky A., Dobrić I. Occurrence of hereditary bullous epidermolyses in Croatia. Pediatr Dermatol. 1990; 7 (2): 108-110.

7. Kho Y.C., Rhodes L. M., Robertson S.J. et al. Epidemiology of epidermolysis bullosa in the antipodes: the Australasian Epidermolysis Bullosa Registry with a focus on Herlitz junctional epidermolysis bullosa. Arch Dermatol 2010; 146: 635-640.

8. McKenna K., Walsh M., Bingham E. Epidermolysis bullosa in Northern Ireland. Br J Dermatol. 1992; 127 (4): 318-321.

9. Horn H.M., Priestley G.C., Eady R.A., Tidman M.J. The prevalence of epidermolysis bullosa in Scotland. Br J Dermatol 1997; 136 (4): $560-564$.

10. Кубанов А. А., Альбанова В.И., Карамова А. Э., Чикин В. В., Мелехина Л.Е., Богданова Е. В. Распространенность врожденного буллезного эпидермолиза у населения Российской Федерации. Вестник дерматологии и венерологии 2015; 3: 21—30. [Kubanov A. A., Albanova V.I., Karamova A.E., Chikin V.V., Melekhina L.Ye., Bogdanova Ye.V. Prevalence of hereditary epidermolysis bullosa in the Russian Federation Vestnik Dermatologii i Venerologii 2015; 3: 21—30 (Russia).]

11. Кубанов А.А., Карамова А.Э., Чикин В.В., и др. Эпидемиология и состояние оказания медицинской помощи больным врожденным буллезным эпидермолизом в Российской Федерации. Вестник Российской академии медицинских наук. 2018; 73 (6): 420-430. [Kubanov A. A., Karamova A.A., Chikin V.V., Bogdanova E. V., Monchakovskaya E.S. Epidemiology and Providing of Healthcare for Patients with Inherited Epidermolysis Bullosa in the Russian Federation Annals of the Russian academy of medical sciences 2018; 73 (6): 420—430 (Russia).]

12. Has C., Nyström A., Saeidian A.H., BrucknerTuderman L., Uitto J. Epidermolysis bullosa: Molecular pathology of connective tissue components in the cutaneous basement membrane zone. Matrix Biol 2018; 7172: 31329 .

13. Has C., Fischer J. Inherited epidermolysis bullosa: New diagnostics and new clinical phenotypes. Exp Dermatol. 2018; 00: 1-7.

14. Lin Z., Li S., Feng C. et al. Stabilizing mutations of KLHL24 ubiquitin ligase cause loss of keratin 14 and human skin fragility. Nat Genet 2016: 48: 1508-1516.

15. Lee J.Y.W., Liu L., Hsu C.-K. et al. Mutations in KLHL24 Add to the Molecular Heterogeneity of Epidermolysis Bullosa Simplex. J Invest Dermatol 2017: 137: 1378-1380.

16. He Y., Maier K., Leppert J. et al. Monoallelic Mutations in the Translation Initiation Codon of KLHL24 Cause Skin Fragility. Am J Hum Genet 2016: 99: 1395-1404

17. Kim S., Coulombe P.A. Intermediate filament scaffolds fulfill mechanical, organizational, and signaling functions in the cytoplasm. Genes Dev 2007; 21: 1581-97.

18. Vahidnezhad H., Youssefian L., Saeidian A.H. et al. Recessive mutation in tetraspanin CD151 causes Kindler syndrome-like epidermolysis bullosa with multi-systemic manifestations including nephropathy. Matrix Biol 2018; 66: 22-33.

19. Gostyńska K. B., Nijenhuis M., Lemmink H. et al. Mutation in exon 1a of PLEC, leading to disruption of plectin isoform 1a, causes autosomal-recessive skin-only epidermolysis bullosa simplex. Hum Mol Genet. 2015; 24 (11): 3155-62.

20. Liu L., Dopping-Hepenstal P.J., Lovell P.A. et al. Autosomal recessive epidermolysis bullosa simplex due to loss of BPAG1-e expression. J Invest Dermatol 2012; 132: 742-744.

21. Takeichi T., Nanda A., Liu L. et al. Founder mutation in dystonin-e underlying autosomal recessive epidermolysis bullosa simplex in Kuwait. $\mathrm{Br}$ J Dermatol 2015; 172 (2): 527-31.

22. Turcan I., Pasmooij A.M.G., Gostyński A. et al. Epidermolysis Bullosa Simplex Caused by Distal Truncation of BPAG1-e: An Intermediate
Generalized Phenotype with Prurigo Papules. J Invest Dermatol 2017; 137 (10): $2227-30$.

23. He Y., Leppert J., Steinke H. et al. Homozygous Nonsense Mutation and Additional Deletion of an Amino Acid in BPAG1e Causing Mild Localized Epidermolysis Bullosa Simplex. Acta Derm Venereol 2017; 97 (5): 657-9.

24. Cappuccio G., Pinelli M., Torella A. et al. Expanding the phenotype of DST-related disorder: a case report suggesting a genotype/phenotype correlation. Am J Med Genet A 201; 173 (10): 2743—6.

25. McGrath J.A., Stone K. L., Begum R. et al. Germline Mutation in EXPH5 Implicates the Rab27B Effector Protein Slac2-b in Inherited Skin Fragility. Am J Hum Genet 2012; 91: 1115-1121.

26. Pigors M., Schwieger-Briel A., Leppert J. et al. Molecular heterogeneity of epidermolysis bullosa simplex: contribution of EXPH5 mutations. J Invest Dermatol 2014; 134: 842-845.

27. Malchin N., Sarig 0., Grafi-Cohen M. et al. A novel homozygous deletion in EXPH5 causes a skin fragility phenotype. Clin Exp Dermatol 2016; 41: 915-918.

28. Rashidghamat E., Ozoemena L., Liu L., McGrath J.A., Martinez A.E., Mellerio J.E. Mutations in EXPH5 (exophilin-5) underlie a rare subtype of autosomal recessive epidermolysis bullosa simplex. Br J Dermatol 2016; 174 (2): 452-453.

29. Has C., Sparta G., Kiritsi D. et al. Integrin alpha3 mutations with kidney, lung, and skin disease. N Engl J Med 2012; 366: 1508_14.

30. Nicolaou N., Margadant C., Kevelam S. H. et al. Gain of glycosylation in integrin alpha3 causes lung disease and nephrotic syndrome. J Clin Invest 2012; 122: 4375-87.

31. Yalcin E.G., He Y., Orhan D., Pazzagli C., Emiralioglu N., Has C. Crucial role of posttranslational modifications of integrin $\alpha 3$ in interstitial lung disease and nephrotic syndrome. Hum Mol Genet 2015; 24: 3679-88.

32. Lovric S., Fang H., Vega-Warner V. et al. Rapid detection of monogenic causes of childhood-onset steroid-resistant nephrotic syndrome. Clin. J. Am. Soc. Nephrol. 2014; 9: 1109_1116.

33. Shukrun R., Vivante A., Pleniceanu 0. et al. A Human Integrin- $\alpha 3$ Mutation Confers Major Renal Developmental Defects. PLoS ONE 2014; 9 (3): e90879.

34. Fine J.D. Inherited epidermolysis bullosa. Orphanet $\mathrm{J}$ Rare Dis 2010; 5-12

35. Fine J.D., Johnson L. B., Weiner M. et al. Pseudosyndactyly and musculoskeletal deformities in inherited epidermolysis bullosa (EB): experience of the National EB Registry, 1986-2002. J Hand Surg (British and European Volume) 200; 30B: 14—22.

36. Fine J.D., Mellerio J. Extracutaneous manifestations and complications of inherited epidermolysis bullosa: Part I. Other organs. J Am Acad Dermatol 2009; 61: 367-384.

37. Fine J.D., Mellerio J. Extracutaneous manifestations and complications of inherited epidermolysis bullosa: Part II. Other organs. J Am Acad Dermatol 2009; 61: 387-402.

38. Fine J.D., Johnson L. B., Weiner M., Suchindran C. Cause-specific risks of childhood death in inherited epidermolysis bullosa. J Pediatr 2008; 152: 276-280.

39. Zidorio A.P. C., Dutra E. S., Leão D. O.D., Costa I. M.C. Nutritional aspects of children and adolescents with epidermolysis bullosa: Literature review. An. Bras. Dermatol. 2015; 90: 217-223.

40. Fine J.D., Johnson L.B., Weiner M., Li K.P., Suchindran C. Epidermolysis bullosa and the risk of life-threatening cancers: the national EB registry experience, 1986-2006. J Am Acad Dermatol 2009; 60: 203-211.

41. Mellerio J.E., Robertson S.J., Bernardis C., Diem A., Fine J.D., George R., et al. Management of cutaneous squamous cell carcinoma in patients with epidermolysis bullosa: best clinical practice guidelines. $\mathrm{Br} \mathrm{J}$ Dermatol 2016; 174: 56-67.

42. Fine J.D., Eady R. A., Bauer E. A. et al. The classification of inherited epidermolysis bullosa (EB): report of the third international consensus 
meeting on diagnosis and classification of EB. J Am Acad Dermatol 2008; 58: $931-950$.

43. Eady R.A. J., Dopping-Hepenstal P.J.C. Transmission electron microscopy for the diagnosis of epidermolysis bullosa. Dermatol Clin 2010; 28 (2): $211-222$.

44. Rao R., Mellerio J., Bhogal B.S., Groves R. Immunofluorescence antigen mapping for hereditary epidermolysis bullosa. Indian J Dermatol Venereol Leprol 2012; 78: 692-7.

45. Takeichi T., Nanda A., Liu L. et al. Impact of next generation se-quencing on diagnostics in a genetic skin disease clinic. ExpDermatol 2013; 22: 825-831.

46. He Y., Balasubramanian M., Humphreys N. et al. Intronic ITGA3 Mutation Impacts Splicing Regulation and Causes Interstitial Lung Disease, Nephrotic Syndrome and Epidermolysis Bullosa. J Invest Dermatol 2016; 136: 1056-1059.

47. Denyer J., Pillay E., Clapham J. Best practice guidelines for skin and wound care in epidermolysis bullosa. An International Consensus. Wounds International 2017.

48. El Hachem M., Zambruno G., Bourdon-Lanoy E. et al. Multicentre consensus recommendations for skin care in inherited epidermolysis bullosa. Orphanet Journal of Rare Diseases 2014; 9: 76.

49. Woo K. Y. Exploring the effects of pain and stress on wound healing. Adv. Skin Wound Care 2012; 25: 38—44.

50. Kiecolt-Glaser J., Marucha P., Malarkey W., Mercado A., Glaser R. Slowing of wound healing by psychological stress. Lancet 1995; 346 (8984): 1194-6.

51. Hwang S. J.E., Daniel B., Fergie B., Davey J., Murrell D. F. Prevalence of anemia in patients with epidermolysis bullosa registered in Australia. IJWD. 2015; 1 (1): 37-40.

52. Haynes L. Clinical practice guidelines for nutritional support in children with epidermolysis bullosa including THINC (Tool to Helpldentify Nutritional Compromise in EB). London: Great Ormond Street Hospital; 2007. 36 p.

53. Zidorio A.P., Dutra E.S., Leão D. O., Costa I.M. Nutritional aspects of children and adolescents with epidermolysis bullosa: literature review. An Bras Dermatol 2015; 90: 217-223.

54. Pope E., Lara-Corrales I., Mellerio J. et al. A consensus approach to wound care in epidermolysis bullosa. J Am Acad Dermatol 2012; 67: 904-917.

55. Rashidghamat E., McGrath J.A. Novel and emerging therapies in the treatment of recessive dystrophic epidermolysis bullosa. Intractable Rare Dis Res 2017; 6: 6-20.

56. Woodley D. T., Keene D.R., Atha T. et al. Injection of recombinant human type VII collagen restores collagen function in dystrophic epidermolysis bullosa. Nat Med 2004; 10 (7): 693-695.

57. Remington J., Wang X., Hou Y. et al. Injection of recombinant human type VII collagen corrects the disease phenotype in a murine model of dystrophic epidermolysis bullosa. Mol Ther 2009; 17: 26-33.

58. Uitto J., Has C., Bruckner-Tuderman L. Cell-based therapies for epidermolysis bullosa - from bench to bedside. J Dtsch Dermatol Ges; 2012; 10 (11): 803-807.

59. Wong T., Gammon L., Liu L. et al. Potential of fibroblast cell therapy for recessive dystrophic epidermolysis bullosa. J Invest Dermatol 2008; 128: $2179-2189$.

60. Kühl T., Mezger M., Hausser I. et al. Collagen VII Half-Life at the Dermal-Epidermal Junction Zone: Implications for Mechanisms and Therapy of Genodermatoses. J Invest Dermatol 2016; 136 (6): 1116—23.

61. Chen M., Woodley D.T. Fibroblasts as target cells for DEB gene therapy. J Invest Dermatol 2006; 126 (4): 708-710.

62. Sriram G., Bigliardi P.L. and Bigliardi-Qi M. Fibroblast heterogeneity and its implications for engineering organotypic skin models in vitro. Europ J Cell Biol 2015; 94: 483-512.

63. Nagy N., Almaani N., Tanaka A. HB-EGF induces COL7A1 expression in keratinocytes and fibroblasts: possible mechanism underlying allogeneic fibroblast therapy in recessive dystrophic epidermolysis bullosa. J Invest Dermatol 2011; 131 (8): 1771—1774.
64. Petrof G., Martinez-Queipo M., Mellerio J.E., Kemp P., McGrath J.A. Fibroblast cell therapy enhances initial healing in recessive dystrophic epidermolysis bullosa wounds: Results of a randomized, vehicle-controlled trial. Br J Dermatol 2013; 169: 1025-1033.

65. Venugopal S.S., Yan W., Frew J.W. A phase II randomized vehicle-controlled trial of intradermal allogeneic fibroblasts for recessive dystrophic epidermolysis bullosa. J Am Acad Dermatol; 2013; 69 (6): 898-908.

66. Tolar J., Ishida-Yamamoto A., Riddle M. et al. Amelioration of epidermolysis bullosa by transfer of wild-type bone marrow cells. Blood 2009; 113: 1167-74.

67. Wagner J. E., Ishida-Yamamoto A., McGrath J.A. et al. Bone marrow transplantation for recessive dystrophic epidermolysis bullosa.N Engl J Med 2010; 363: 629_39.

68. Tolar J., Wagner J.E. Allogeneic blood and bone marrow cells for the treatment of severe epidermolysis bullosa: repair of the extracellular matrix Lancet 2013; 382 (9899): 1214-1223.

69. Sasaki M., Abe R., Fujita Y., Ando S., Inokuma D., Shimizu H. Mesenchymal stem cells are recruited into wounded skin and contribute to wound repair by transdifferentiation into multiple skin cell type. J Immunol 2008; 180: 2581-7.

70. Petrof G., Lwin S.M., Martinez-Queipo M. et al. Potential of Systemic Allogeneic Mesenchymal Stromal Cell Therapy for Children with Recessive Dystrophic Epidermolysis Bullosa. J Invest Dermatol 2015; 135 : 2319-2321.

71. Takahashi K., Yamanaka S. Induction of pluripotent stem cells from mouse embryonic and adult fibroblast cultures by defined factors. Cell 2006; 126: 663-76.

72. Yu J., Vodyanik M.A., Smuga-Otto K. et al. Induced pluripotent stem cell lines derived from human somatic cells. Science 2007; 318: 1917-20.

73. Hanna J., Wernig M., Markoulaki S. et al. Treatment of sickle cell anemia mouse model with iPS cells generated from autologous skin. Science 2007; 318: 1920-3.

74. Tedesco F. S., Gerli M.F., Perani L. et al. Transplantation of genetically corrected human iPSC-derived progenitors in mice with limb-girdle muscular dystrophy. Sci Transl Med 2012; 4: 140ra89.

75. Mandai M., Watanabe A., Kurimoto Y. et al. Autologous induced stemcell-derived retinal cells for macular degeneration. N Engl J Med 2017; 376: 1038 - 46.

76. Araki R., Uda M., Hoki Y. et al. Negligible immunogenicity of terminally differentiated cells derived from induced pluripotent or embryonic stem cells. Nature 2013; 494: 100—4.

77. Tolar J., Xia L., Riddle M.J. et al. Induced pluripotent stem cells from individuals with recessive dystrophic epidermolysis bullosa. J Invest Dermatol 2011; 131: 848-56.

78. Tolar J., Xia L., Lees C.J. et al. Keratinocytes from induced pluripotent stem cells in junctional epidermolysis bullosa. J Invest Dermatol 2013; 133: 562-5.

79. Jackow J., Titeux M., Portier S. et al. Gene-corrected fibroblast therapy for recessive dystrophic epidermolysis bullosa using a self-inactivating COL7A1 retroviral vector. J Invest Dermatol 2016; 136: 1346-54.

80. Gache Y., Allegra M., Bodemer C., Pisani-Spadafora A., de Prost Y., Ortonne J.P., Meneguzzi G. Genetic bases of severe junctional epidermolysis bullosa presenting spontaneous amelioration with aging. Hum Mol Genet 2001; 10: 2453-2461.

81. Titeux M., Pendaries V., Zanta-Boussif M.A. et al. SIN retroviral vectors expressing COL7A1 under human promoters for ex vivo gene therapy of recessive dystrophic epidermolysis bullosa. Mol Ther 2010; 18: $1509-18$

82. Sebastiano V., Zhen H.H., Haddad B. et al. Human COL7A1-corrected induced pluripotent stem cells for the treatment of recessive dystrophic epidermolysis bullosa. Sci Transl Med 2014; 6: 264 ra163. 
83. Georgiadis C., Syed F., Petrova A. et al. Lentiviral engineered fibroblasts expressing codon-optimized COL7A1 restore anchoring fibrils in RDEB. J Invest Dermatol 2016; 136: 284—92.
84. Hirsch T., Rothoeft T., Teig N. et al. Regeneration of the entire human epidermis using transgenic stem cells. Nature 2017; 551: $327-332$

\section{Информация об авторах}

Алексей Алексеевич Кубанов - чл.-корр. РАН, д.м.Н., просрессор, директор ФГБУ «ГНЦДК» Минздрава России; е-mail: aleх@сnikvi.ru Арфеня Эдуардовна Карамова - к.м.Н., заведующий отделом дерматологии ФГБУ «ГНЦДК» Минздрава России; e-mail: karamova@cnikvi.ru

Екатерина Сергеевна Мончаковская - младший научный сотрудник отдела дерматологии ФГБУ «ГНЦДК» Минздрава России; е-таil: monchakovskaya@cnikvi.ru

\section{Information about the authors}

Alexey A. Kubanov - Dr. Sci. (Med.), Prof., Corresponding Member of the Russian Academy of Sciences, Director, State Research Center of Dermatovenereology and Cosmetology, Ministry of Health of the Russian Federation; e-mail: alex@cnikvi.ru

Arfenya E. Karamova - Cand. Sci. (Med)., Head of the Department of Dermatology, State Research Center of Dermatovenereology and Cosmetology, Ministry of Health of the Russian Federation; e-mail: karamova@cnikvi.ru

Ekaterina S. Monchakovskaya - Junior Research Fellow, Department of Dermatology, State Research Center of Dermatovenereology and Cosmetology, Ministry of Health of the Russian Federation; e-mail: monchakovskaya@cnikvi.ru 